\title{
DNS analysis of the effects of combustion on turbulence in a supersonic H2/air jet flow
}

\author{
Yaowei $\mathrm{Fu}^{\mathrm{a}, \mathrm{b}}$, Changping $\mathrm{Yu}^{\mathrm{a}, \mathrm{b}}$, Zheng Yan ${ }^{\mathrm{a}, \mathrm{b}}$, Xinliang $\mathrm{Li}^{\mathrm{a}, \mathrm{b}, *}$ \\ a LHD, Institute of Mechanics, Chinese Academy of Sciences, Beijing 100190, China \\ b School of Engineering Science, University of Chinese Academy of Sciences, Beijing 100049, China
}

\section{A R T I C L E I N F O}

\section{Article history:}

Received 21 February 2019

Received in revised form 27 June 2019

Accepted 23 August 2019

Available online 29 August 2019

\section{Keywords:}

Supersonic combustion

Turbulence jet

Direct numerical simulations

Coherent structures

\begin{abstract}
A B S T R A C T
In this paper, the effects of combustion on turbulence features are investigated by direct numerical simulation (DNS) of supersonic round turbulent hydrogen jet flows. Two DNS cases are conducted: the one is combustion hydrogen/air jet and the other is non-combustion jet with the same components as a comparison case. In the DNS, the supersonic jet consists of $85 \%$ hydrogen and $15 \%$ nitrogen in volume with temperature of $305 \mathrm{~K}$ and jet velocity of $900 \mathrm{~m} / \mathrm{s}$. The ambient air velocity is $20 \mathrm{~m} / \mathrm{s}$ and temperature is $1150 \mathrm{~K}$. The Reynolds numbers based the jet exit diameter and hydrogen jet velocity both are 22000 and the jet Mach numbers both are 1.2. The DNS results show that occurrence of combustion significantly delays the transition of the jet flow, and results in a $38 \%$ decrease in the peak of turbulent kinetic energy, compared with the non-combustion jet flow. The decreasing of the Reynolds number due to the heat release is considered as the main reason. Compared with the non-combustion jet flow, the combustion jet flow has more complete coherent vortex rings in the early stage. In addition, the positive and minus energy fluxes of combustion jet flow are always larger than that of non-combustion jet flow.
\end{abstract}

(C) 2019 Elsevier Masson SAS. All rights reserved.

\section{Introduction}

Supersonic combustion ramjet (Scramjet) is considered to be one of the best propulsive options for supersonic or hypersonic vehicles [1]. Compared with rocket engines, scramjets do not require additional oxidizer tanks [2]. Compared with turbofan engines, scramjets have smaller total pressure loss for supersonic or hypersonic flights. Therefore, the scramjet is the most effective engine cycle for high speed aircraft. From 1958, when Weber and MacKey [3] demonstrated that combustion could take place in a supersonic airflow, to the first successful flight of the X-43 included an integrated hypersonic airframe-scramjet engine in 2004 [4], the development of scramjet has been somewhat slow. One of the major obstacles for the scramjet engine is the less understanding about the mechanism of supersonic turbulent combustion.

Because of the large gradients of species, the short residence time, the very high temperature so that the real gas effects have to be considered and the closely coupled interaction between mixing and chemical kinetics, the physical processes in the scramjet are more intricate than in most of the other propulsion systems. Therefore, it is almost impossible to explore the interac-

\footnotetext{
* Corresponding author at: LHD, Institute of Mechanics, Chinese Academy of Sciences, Beijing 100190, China.

E-mail address: lixl@imech.ac.cn (X. Li).
}

tion mechanism between turbulence and combustion by theoretical method. Flight test is a particularly effective research method. However, the cost of flight experiment is incredibly expensive so that no country or organization can afford it all the time. Thanks to the rapid development of computer technologies and computing methods, computational fluid dynamics (CFD) is playing an increasingly important role in the field of turbulent reacting flows. There are mainly three approaches used in the turbulent reacting flows: Reynolds-averaged Navier-Stokes equations (RANS), large eddy simulation (LES) and direct numerical simulation (DNS). RANS method, mainly used in practical industrial simulation, solves the mean values of all quantities and the computational cost of this method is minimal. In LES, the large scales are resolved and the effects of smaller scales are modeled by subgrid closure rules. Although LES can provide a time fluctuation at the frequency scale allowed by the grid-scale cutoff, it still filters the small fluctuations. DNS technique is used to resolve all scales of turbulence, and it does not contain any approaches errors associated with turbulence dynamics modeling. However, because of expensive computing cost, DNS is still limited to simple academic flows [5]. Because the scale of interaction between turbulence and combustion is very small, DNS is a good choice to study the mechanism of turbulence combustion interaction.

The mechanism of interaction between combustion and turbulence is a hot topic in recent years. The effects between turbulence 
and combustion are reciprocal. Up to now, most studies have focused on the effects of turbulence on combustion. Saqr [6] numerically investigated the turbulence-combustion interaction in nonpremixed methane-air flames and found that gradually increasing the turbulence intensity of free stream could significantly reduce the reaction zone and even leaded to flame extinction. Lu [7], Jin $[8,9]$ and Luo [10] performed DNS to explore the performance of turbulent combustion models and analyze the flame structures. Using direct numerical simulation data of turbulent $\mathrm{H} 2$-air flames, Wacks [11] investigated the distributions of flow topologies in different regimes of premixed turbulent combustion.

Gottenberg [12] experimentally studied the effects of turbulence on unburnt hydrocarbons found in the chamber and exhaust reservoir. Barlow [13] experimentally researched the effects of turbulence on species mass fractions in piloted methane jet flame. Moen's experiment [14] showed that the turbulence produced by obstacles in the path of a freely propagating $\mathrm{CH} 4 /$ air flame in cylindrical geometry had an obvious effect on the flame propagation. Zhou [15] visualized the flame front structures in swirlstabilized lean premixed methane/air flames by using single-shot planar laser-induced fluorescence method to obtain high quality images. His experimental results show that distributed reactions can be a common combustion mode in the highly turbulent premixed jet flames. Li [16] studied the influence of turbulence intensity on flame local propagation characteristics and flame local structural characteristics and showed that the sensitivity of the local propagation speed of flame to its local characteristics reduced with the increase of the turbulence intensity.

However, to the author's knowledge, there are few researches to devote to the effects of combustion on turbulence. Combustion has two different effects on turbulence: on the one hand, the heat release of combustion decreases the local Reynolds number and then weakens turbulence by increasing the viscosity and decrease the density of fluid; on the other hand, combustion expands fluid so that the turbulence intensity is enhanced. The mechanism of the combustion effects on turbulent is far from clear, and it is also necessary to be studied.

Motivated by this point, we conduct two direct numerical simulations of supersonic round turbulent hydrogen jets to investigate the effects of the chemical reactions on the turbulence characteristics. As a simple but very meaningful physical model, turbulent jet has been studied in detail by many researchers. Many characteristics of turbulent jet, such as decay rate of centerline streamwise mean velocity [17-20], spreading rate of half-velocity jet width [21-24] and self-similarity of streamwise mean velocity profiles $[25,26]$, have been understood. Many factors affecting the characteristics of turbulent jet have also been researched, such as the compressibility [27], the different inflow conditions [28], the different Reynolds numbers [26] and the nozzles of complex geometry $[29,30]$. In this paper, we put our attentions on the change of turbulence characteristics when combustion occurs. The specific problem setting and code validation are introduced in Section 2. Then in Section 3, some results will be discussed. Finally, some concise conclusions obtained from the numerical study are addressed in the Section 4.

\section{Governing equations and numerical methods}

Two direct numerical simulations of three dimensional spatially-developing supersonic round turbulent hydrogen jets are conducted, one case contains chemical reaction, and the other closes chemical reaction, as a comparison case. High order finite difference solver developed by our group for the chemical reacting flows, named as OpenCFD-Comb, is used for the DNS cases. The inlet condition of the round jet flow is the same as that in references [7-10], namely the supersonic jet consists of $85 \%$ hydrogen and $15 \%$ nitrogen in volume with a temperature of $305 \mathrm{~K}$ and a jet velocity of $900 \mathrm{~m} / \mathrm{s}$. The ambient air (co-flow) velocity is $20 \mathrm{~m} / \mathrm{s}$ and the temperature is $1150 \mathrm{~K}$. The jet exit diameter, denoted $\mathrm{D}$, is $1.44 \mathrm{~mm}$. The Reynolds numbers based on the jet exit diameter and hydrogen jet velocity for the both cases are 22000 and the jet Mach number for the both cases are $M a=U_{j} / a_{j}=1.2$. Here, $U_{j}$ is the hydrogen jet velocity and $a_{j}$ is the sound velocity of jet exit.

The conservative three-dimensional compressible Navier-Stokes equations together with the conservation equations of species are given as:

$$
\begin{aligned}
& \frac{\partial \rho}{\partial t}+\frac{\partial\left(\rho u_{i}\right)}{\partial x_{i}}=0 \\
& \frac{\partial\left(\rho u_{i}\right)}{\partial t}+\frac{\partial\left(\rho u_{i} u_{j}\right)}{\partial x_{j}}=\frac{-\partial P}{\partial x_{i}}+\frac{\partial \tau_{i j}}{\partial x_{j}} \\
& \frac{\partial E}{\partial t}+\frac{\partial\left[(E+P) u_{j}\right]}{\partial x_{j}}=\frac{\partial\left[u_{i} \tau_{i j}+q_{j}\right]}{\partial x_{j}}+Q_{s} \\
& \frac{\partial \rho_{k}}{\partial t}+\frac{\partial\left(\rho_{k} u_{i}\right)}{\partial x_{i}}=\frac{\partial}{\partial x_{i}}\left[\rho D_{k m} \frac{\partial Y_{k}}{\partial x_{i}}\right]+\hat{\omega}_{k}
\end{aligned}
$$

where $\rho$ is the density of mixture, $u_{i}$ is the fluid velocity, $P$ is the static pressure, $\tau_{i j}$ is the viscous stress tensor, $Y_{k}$ is the mass fraction of species $k, \rho_{k}$ is the density of species $k$ and can be expressed as $\rho_{k}=\rho Y_{k}$. E and $Q_{s}$ is the total energy per unit volume and heat release rate, respectively. $\hat{\omega}_{k}$ is the production rate of species $k . D_{k m}$ is the mixture diffusion coefficient of species $k$. In present simulations, standard forms of polynomial fits used by the NASA [31] are employed to calculate the thermodynamic properties.

$$
\begin{aligned}
& \frac{C_{p k}}{R_{0}}=a_{1 k}+a_{2 k} T+a_{3 k} T^{2}+a_{4 k} T^{3}+a_{5 k} T^{4} \\
& \frac{H_{k}}{R_{0} T}=a_{1 k}+\frac{1}{2} a_{2 k} T+\frac{1}{3} a_{3 k} T^{2}+\frac{1}{4} a_{4 k} T^{3}+\frac{1}{5} a_{5 k} T^{4}+\frac{a_{6 k}}{T} \\
& \frac{S_{k}}{R_{0}}=a_{1 k} \ln T+a_{2 k} T+\frac{1}{2} a_{3 k} T^{2}+\frac{1}{3} a_{4 k} T^{3}+\frac{1}{4} a_{5 k} T^{4}+a_{7 k}
\end{aligned}
$$

Here, $C_{p k}$ is the molar heat capacity at constant pressure of species $k, H_{k}$ is the molar enthalpy of species $k, S_{k}$ is the species molar entropy and $R_{0}$ is the universal gas constant.

In order to accurately obtain the gas-phase species transport properties, the same methods as CHEMKIN [32] are used, although those approaches are computational expensively. For the viscosity coefficient and the thermal conductivity coefficient of pure species $k$, the form of fitting polynomial is respectively:

$$
\begin{aligned}
& \ln \mu_{k}=\sum_{n=1}^{N} b_{n, k}(\ln T)^{n-1} \\
& \ln \lambda_{k}=\sum_{n=1}^{N} c_{n, k}(\ln T)^{n-1}
\end{aligned}
$$

The fitting polynomial for each pair of binary diffusion coefficient is:

$$
\ln D_{k j}=\sum_{n=1}^{N} d_{n, k j}(\ln T)^{n-1}
$$

The Wilke formula [33] is used to compute the mixture viscosity coefficient and thermal conductivity coefficient.

$\mu=\sum_{k=1}^{N} \frac{X_{k} \mu_{k}}{\sum_{j=1}^{N} X_{j} \phi_{k j}}$ 

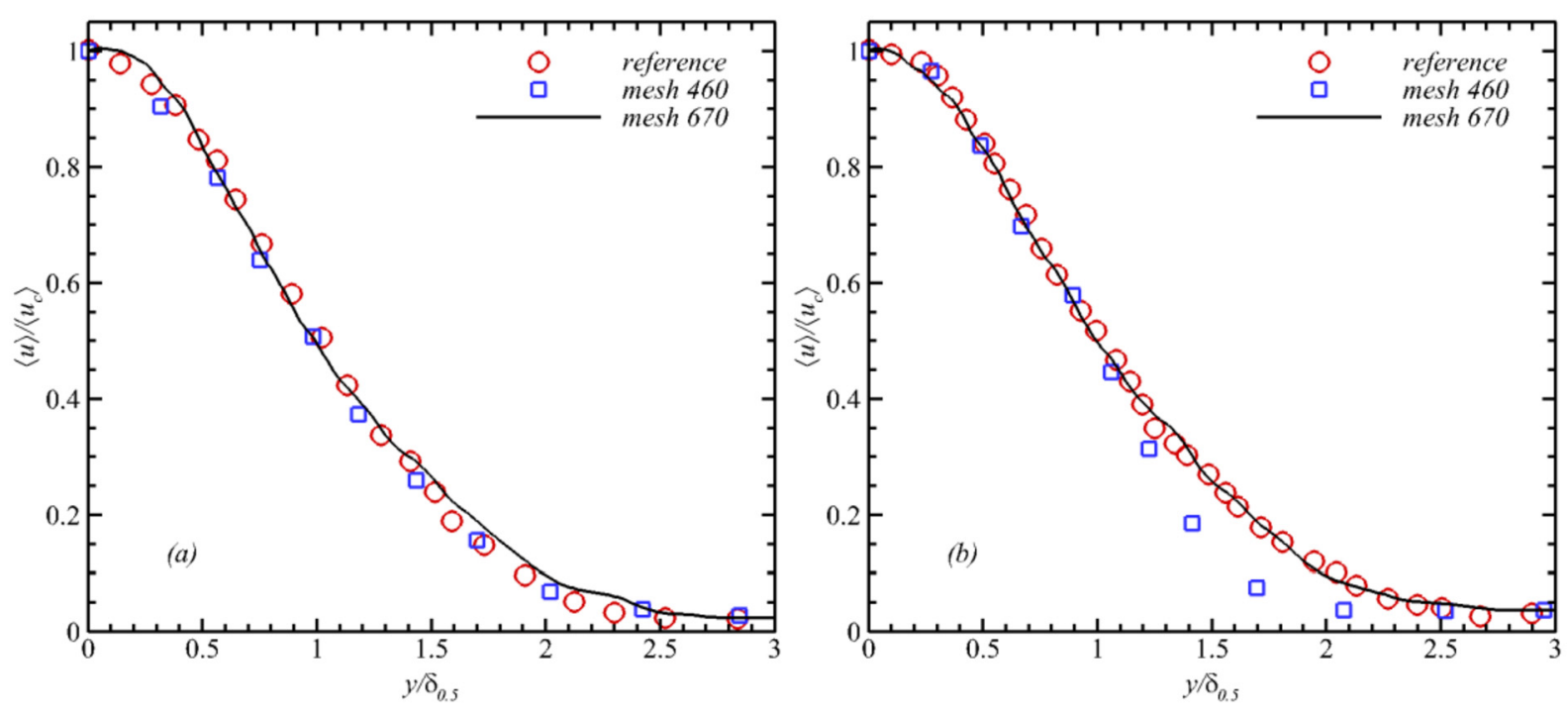

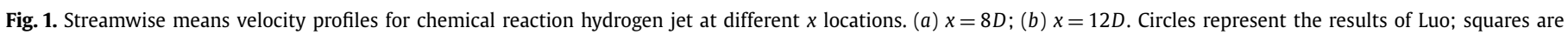
the results of coarse mesh; lines are the results of fine mesh.

$$
\begin{aligned}
& \phi_{k j}=\frac{1}{\sqrt{8}}\left(1+\frac{W_{k}}{W_{j}}\right)^{-\frac{1}{2}}\left(1+\left(\frac{W_{j}}{W_{k}}\right)^{\frac{1}{4}}\left(\frac{\mu_{k}}{\mu_{j}}\right)^{\frac{1}{4}}\right)^{2} \\
& \lambda=\frac{1}{2}\left(\sum_{k=1}^{N} X_{k} \lambda_{k}+\frac{1}{\sum_{k=1}^{N} X_{k} \lambda_{k}}\right)
\end{aligned}
$$

The mixture diffusion coefficient for species $k$ is computed as [34]:

$$
D_{k m}=\frac{1-Y_{k}}{\sum_{j \neq k}^{N} X_{j} / D_{j k}}
$$

where $X_{k}$ is the mole fraction of species $k . W_{k}$ is the molecular weight of species $k . N$ is the total number of species.

The sixth-order monotonicity-preserving optimized scheme (OMP6) [35] is employed to discrete the convective terms and the eighth-order centered difference method is adopted for the viscous terms. It is worth noting that all the computing settings are identical for the chemical reaction and non-chemical-reaction jet flow to avoid difference duo to different numerical methods. The thirdorder Runge-Kutta approach is used for the time advancement. For the reaction case, in order to overcome the stiffness of reaction source terms, the Strang time-splitting scheme [36,37] is used.

$L(\Delta t)=L_{1}\left(\frac{\Delta t}{2}\right)\left[\sum_{m=1}^{M} L_{2}\left(\frac{\Delta t}{M}\right)\right] L_{1}\left(\frac{\Delta t}{2}\right)$

Here, $L_{1}$ is the time advancing operator for flow dynamics, and $L_{2}$ is the time advancing operator for chemical reaction source terms. $\Delta t$ is the time step, and it is set as $10^{-8} \mathrm{~s}$ (dimensionless time step $\left.\overline{\Delta t}=\Delta t /\left(D / U_{j}\right)=6.25 \times 10^{-3}\right)$ in the DNS; $M$ is the number of sub-time steps for chemical reaction source terms in each time step. Here we set $M=5$.

In order to capture the intricate physical phenomenon, such as the transition of laminar to turbulence, the autoignition of the hydrogen and the entrainment phenomenon, a large enough computation domain is designed in a Cartesian coordinate system as $0 \leq x \leq 15 D,-7 D \leq y, z \leq 7 D(0 \mathrm{~mm} \leq x \leq 21.6 \mathrm{~mm}$, $-10 \mathrm{~mm} \leq y, z \leq 10 \mathrm{~mm}$ ). A sponge layer, approximately $13 \mathrm{D}$, with nonuniform coarse grids is added at the outlet region to avoid the effect of reflected waves. Two different grids are used for comparison. The total structured nodes are $460 \times 399 \times 399$ and $670 \times 459 \times 459$, respectively. When computing the chemical reaction, because multi-step chemistry predicts intricate details of the combustion process better than single step chemistry [38], so the detailed $\mathrm{H} 2 / \mathrm{O} 2$ chemical kinetic mechanism of Li [39], which includes 9 species and 19 reactions, is employed.

Two streamwise mean velocity profiles for chemical reaction hydrogen jet at different $x$ locations are shown in Fig. 1. The streamwise mean velocity of different $y$ positions, denoted $\langle u\rangle$, are normalized with the local centerline streamwise mean velocity $\left\langle u_{c}\right\rangle$ and the distances from the centerline are scaled with the half-velocity jet width $\delta_{0.5}$, which is the distance between the centerline and a point where the local mean velocity is half of the local centerline mean velocity. Fig. 1 indicates that the current DNS results are in good agreement with the results of Luo's simulation [10], which validate the current DNS.

\section{Results and discussions}

Two instantaneous images of the vorticity magnitude, $|\Omega|=$ $|\nabla \times \vec{V}|$, are displayed in Fig. 2 for combustion jet flow and noncombustion jet flow. For non-combustion jet flow, Fig. 2(a), the flow remains laminar in the region of $x<2.2 D$. Further downstream, because of the Kelvin-Helmholtz instability mechanism, the coherent vortex ring structures roll up. Under the action of three-dimensional instability, after the region of $x>3.5 D$, these coherent vortex ring structures start to merge and breakup and then the flow becomes fully turbulent gradually. In the case of combustion jet flow, Fig. $2(b)$, in the region of $x<1.5 D$, the flow is laminar. Then the coherent vortex rings roll up. After the region of $x>4.5 D$, the flow starts to transition to turbulence. Compared with the non-combustion jet flow, the combustion jet has more complete coherent vortex rings in the initial stage.

Fig. 3 shows the development process of three-dimensional vortex structures of present two jet flows. These vortex structures are identified by Q-criterion and flood by temperature, which is defined as $Q=\frac{1}{2} \frac{\partial u_{i}}{\partial x_{j}} \frac{\partial u_{j}}{\partial x_{i}}$. It is observed that in the initial stage of non-combustion jet flow the vortex rings are helical. However, the vortex rings of combustion jet flow in the initial stage are axisymmetric. It can be found from Fig. 3 that the vortex rings begin to breakdown downstream $x=3.5 D$ for non-combustion jet flow and downstream $x=4.5 \mathrm{D}$ for combustion jet flow, which means that the transition is significantly delayed when combustion occurs. Compared with non-combustion one, there are less vortex filament structures in the combustion jet, and the scales of the vortex filaments are more coarse. The main reason is that the heat 

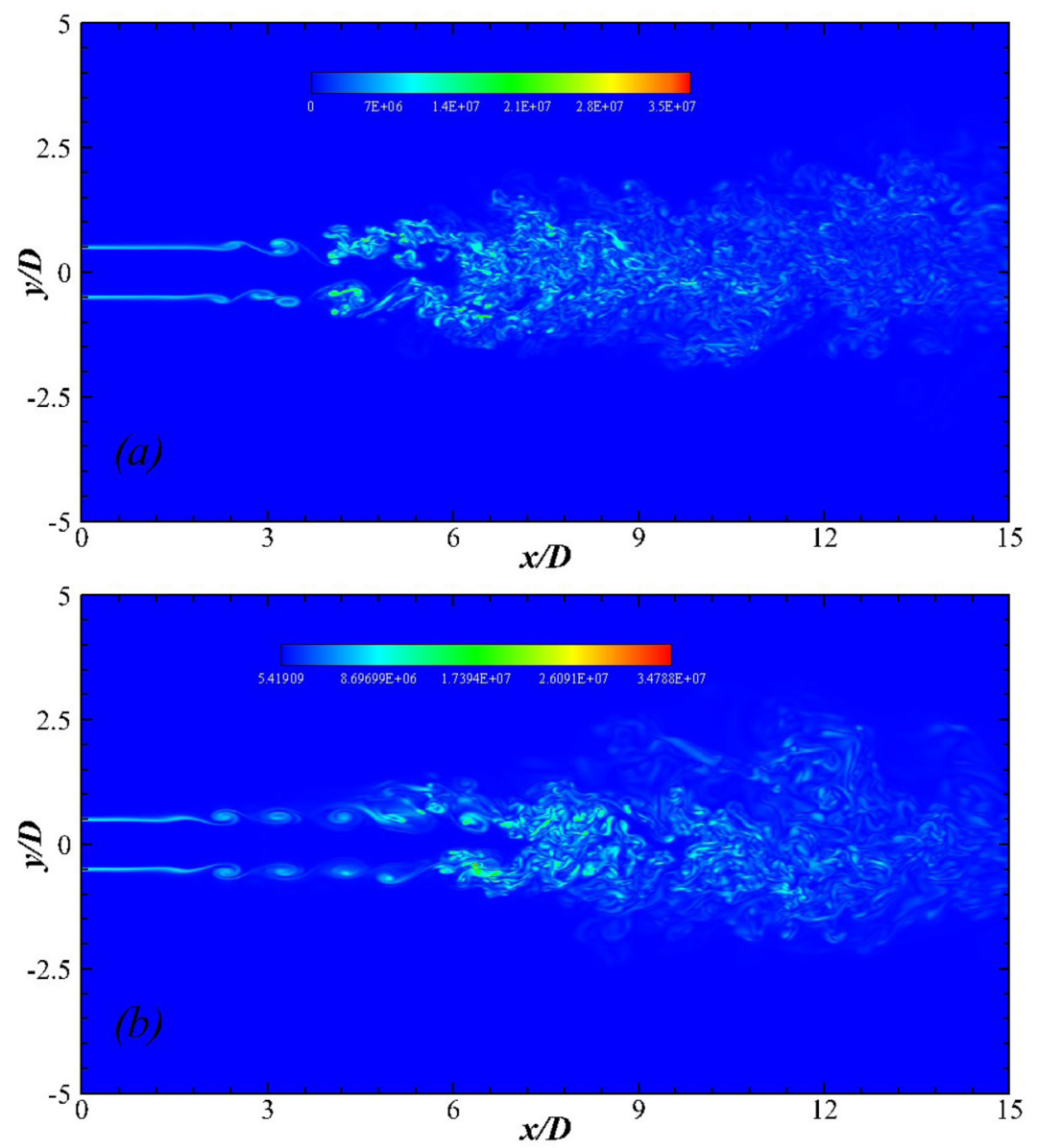

Fig. 2. Magnitude of instantaneous vorticity field $|\Omega|=|\nabla \times \vec{V}|$ at the plane of $z=0$. (a) Non-combustion jet flow; (b) combustion jet flow. (For interpretation of the colors in the figure(s), the reader is referred to the web version of this article.)
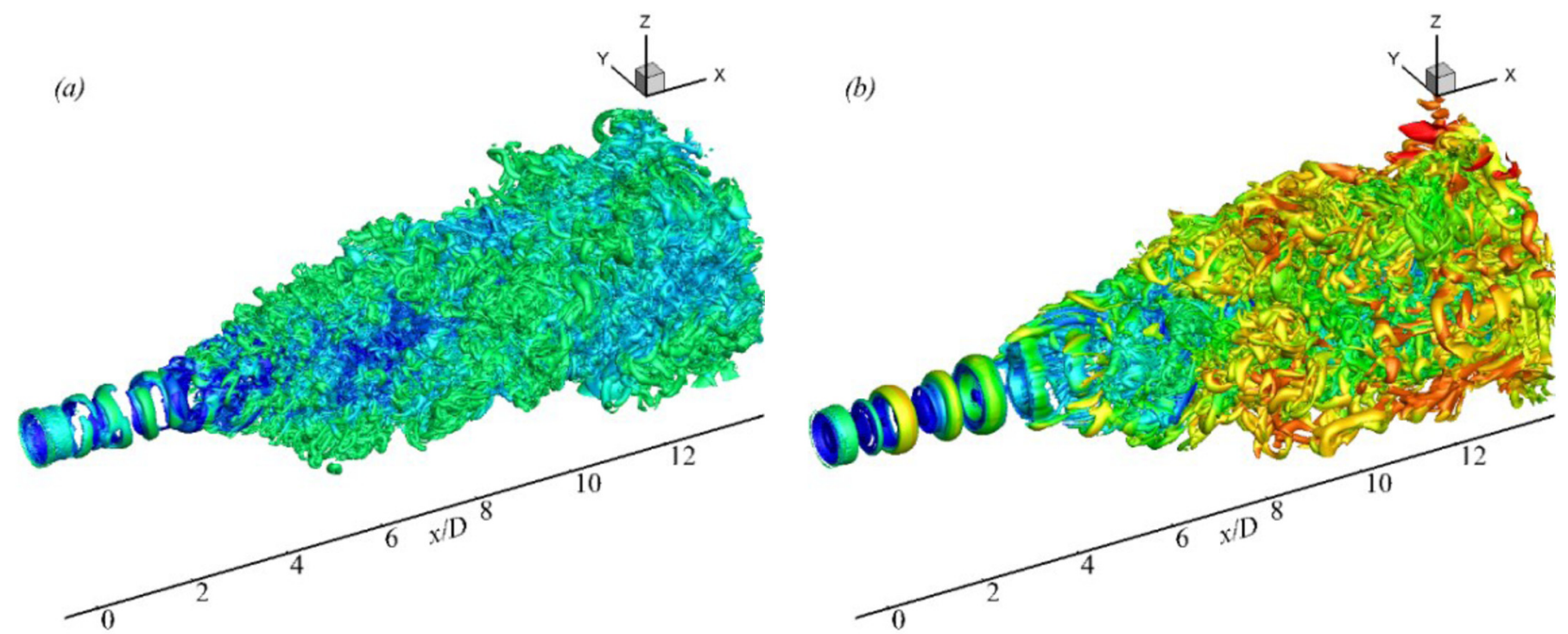

Fig. 3. Vortex structures identified by Q-criterion using the same value of $Q$ flood by temperature. (a) Non-combustion jet flow, $Q=1 \times 10^{10} \mathrm{~s}^{-2}$; $(b)$ combustion jet flow, $Q=1 \times 10^{10} \mathrm{~s}^{-2}$.

release of combustion decreases the actual Reynolds number of the combustion case, leading to this phenomenon.

Fig. 4 and Fig. 5 display instantaneous snapshots of $\left(\omega_{y}^{2}+\omega_{z}^{2}\right)^{0.5}$ at nine different axial cross sections downstream of the jet exit with the non-combustion jet and combustion jet, respectively. Here $\omega_{y}$ and $\omega_{z}$ are vorticity components in $y$ - and $z$-direction, respec- tively. This quantity can qualitatively represent the change of shear layer. For non-combustion jet flow, the shear layer is fairly circular before $x=2 D$ and then begin to wrinkle. Until $x=4 D$, the shear layer is completely wrinkled. However, for combustion jet flow, the shear layer remains circular before $x=4 D$ and is completely wrinkled after $x=6 D$. This means that combustion jet flow 

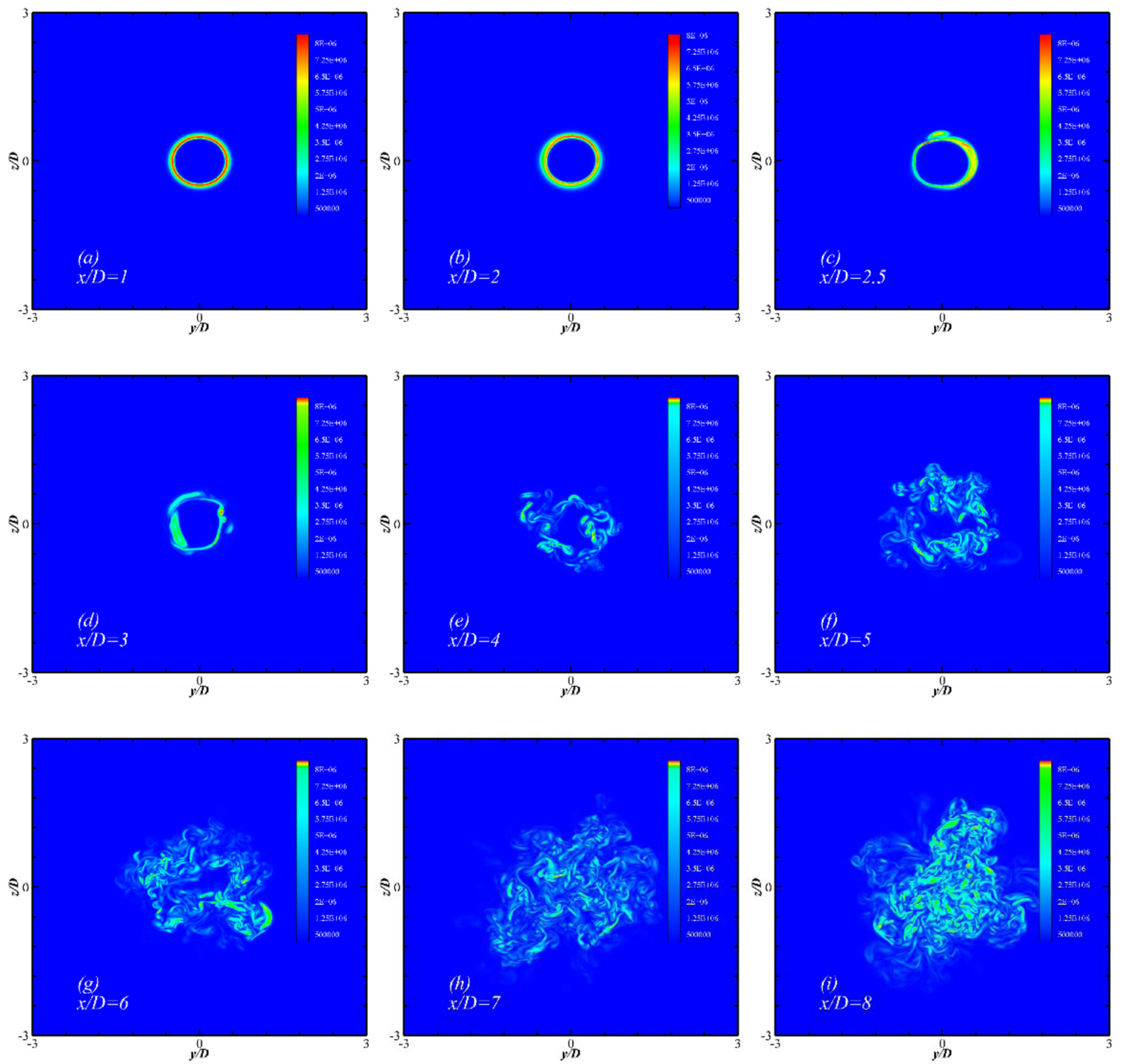

Fig. 4. Instantaneous filed of the $\left(\omega_{y}^{2}+\omega_{z}^{2}\right)^{0.5}$ for non-combustion jet flow at several different axial cross sections, $x / D=1,2,2.5,3,4,5,6,7,8$.

is more stable. In particular, comparing Fig. 4(c) and Fig. $4(d)$ with Fig. 5(e) and Fig. 4( $f)$, it can be found that the process of shear layer instability of combustion jet flow is very different from that of non-combustion jet flow.

The development of local mean Reynolds number, $R e=\left\langle\rho_{l} u_{l} D /\right.$ $\left.\mu_{l}\right\rangle$, and local mean streamwise velocity at the plane of $z=0$ is shown in Fig. 6. Here $\rho_{l}, u_{l}$ and $\mu_{l}$ is local density, local streamwise velocity and local viscosity of fluid, respectively. $\langle\cdot\rangle$ denotes the time average. Fig. 6(a) describes the change of Reynolds number of the fixed normal position $y=0.5 D$ with respect to the coordinate $x$. Fig. 6(b) depicts the evolution of Reynolds number of different $y$ positions at the axial cross section of $x=12 D$. The local mean Reynolds number is closely related to the development of flow instability, the turbulent vortex structure and the evolution of shear layer. In general, the lager the local mean Reynolds num- ber is, the faster the flow instability develops, and the finer the turbulence vortex scale is. It can be found from Fig. 6 that the local mean Reynolds number of combustion jet flow is much smaller than that of non-combustion jet flow. The main reason is that combustion releases large amount of heat so that leads to the increase of the viscosity of fluid and the decrease of the local density.

The variation of turbulence kinetic energy (TKE) along the centerline, $T K E=\frac{1}{2}\left\langle\rho u_{i}^{\prime} u_{i}^{\prime}\right\rangle$, is shown in Fig. 7. The superscript prime represents the fluctuation of Reynolds average. The turbulent kinetic energy is normalized by jet exit velocity and density. It can be seen from Fig. 7 that both TKE lines keep very little value in the initial range of $x<1 D$. As the jet flows downstream, the flow instabilities develop and the turbulent kinetic energy increases rapidly. At the early range $1 D<x<3 D$, TKE of the combustion jet is higher than that of the non-combustion one, and this is 

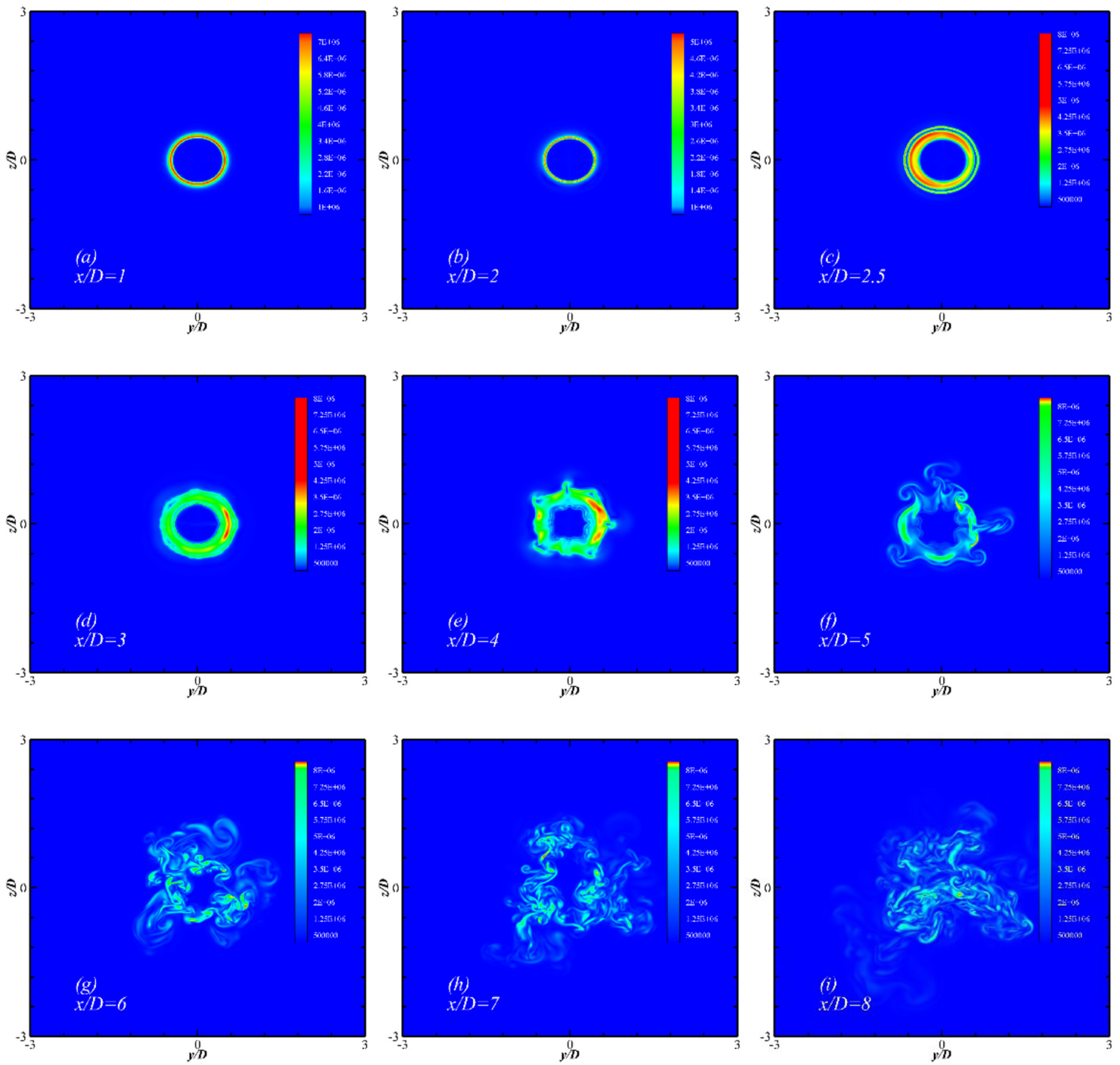

Fig. 5. Instantaneous filed of the $\left(\omega_{y}^{2}+\omega_{z}^{2}\right)^{0.5}$ for combustion jet flow at several different axial cross sections, $x / D=1,2,2.5,3,4,5,6,7,8$.

related with the perturbations due to the thermal expansion of fluids. But at the range $x>3 D$, TKE of combustion jet develops much slower than that of the non-combustion one, and this is related with the lower local Reynolds number of the combustion case. For non-combustion jet flow, the peak value of non-dimensional TKE is 0.039 at the position of $x=6.8 D$. However, for combustion jet flow, the peak value of is 0.024 at the position of $x=8.6 D$. The occurrence of combustion results in a $38 \%$ decrease in the peak of turbulent kinetic energy. It is worth noting that the turbulence kinetic energy of present two jet flows are basically the same in the fully developed turbulent region of $x>10 D$. The main reason is that in the fully developed turbulent region of present combustion jet flow, the effect of TKE decreasing due to lower Reynolds number by heat release is offset by the effect of TKE increasing due to fluid expansion caused by combustion.
In the fully developed turbulent region of present two jet flows, identical regions $(11 D \leq x \leq 12 D,-0.5 D \leq y \leq 0.5 D,-0.5 D \leq z \leq$ 0.5 ) are selected to investigate the characteristics of scale-to-scale transfer of turbulent kinetic energy. The local energy flux is calculated by using a filtering approach. The governing equation of the large-scale kinetic energy can be obtained by applying a filtering operation to the compressible Navier-Stokes equations [40].

$$
\begin{aligned}
& \frac{\partial}{\partial t}\left(\frac{1}{2} \bar{\rho} \tilde{u}_{i}^{2}\right)+\frac{\partial}{\partial x_{j}}\left(\frac{1}{2} \bar{\rho} \tilde{u}_{i}^{2} \tilde{u}_{j}+\bar{P} \tilde{u}_{j}+\tau_{i j} \tilde{u}_{i}\right)=\bar{P} \frac{\partial \tilde{u}_{i}}{\partial x_{i}}-\Pi \\
& \tilde{S}_{i j}=\frac{1}{2}\left(\frac{\partial \tilde{u}_{i}}{\partial x_{j}}+\frac{\partial \tilde{u}_{j}}{\partial x_{i}}\right) \\
& \tau_{i j}=\overline{\rho u_{i} u_{j}}-\bar{\rho} \tilde{u}_{i} \tilde{u}_{j} \\
& \Pi=-\tau_{i j} \tilde{S}_{i j}
\end{aligned}
$$



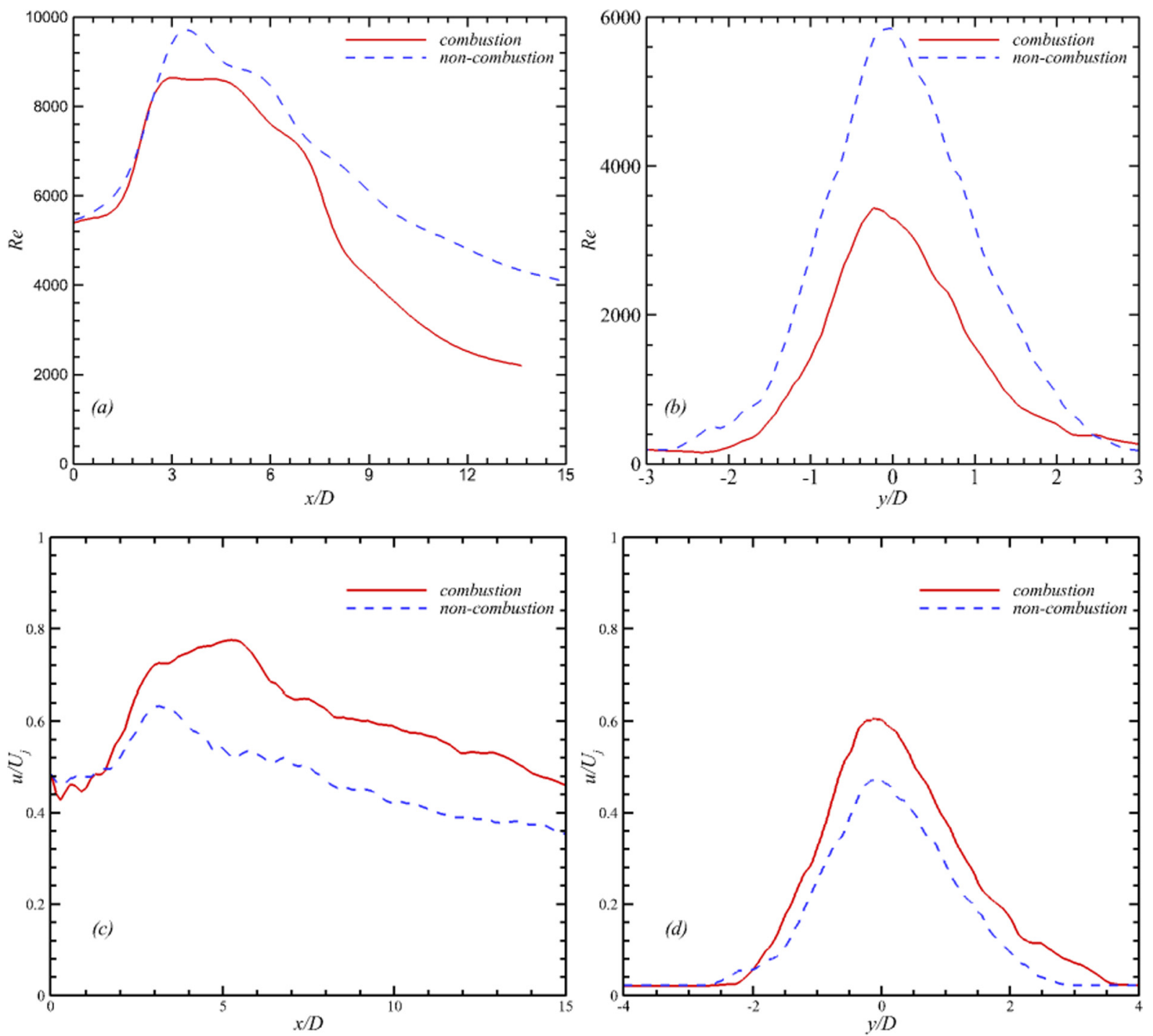

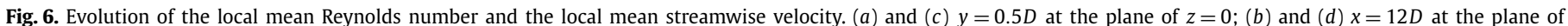
$z=0$.

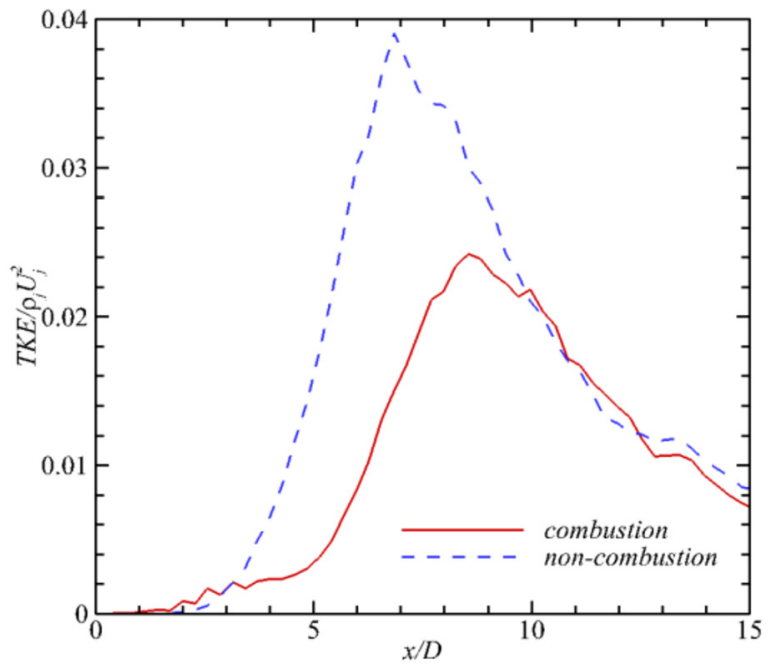

Fig. 7. Evolution of turbulence kinetic energy along the centerline.

Here, a tilde denotes the Favre filtering $\tilde{f}=\bar{\rho} f / \bar{\rho}$ and an overbar represents the low-pass filtering. In this paper, a top-hat filter is used to get coarse-grained filed in present two numerical simulations.

$\bar{a}=\frac{1}{4 n}\left(a_{i-n}+2 \sum_{j=i-n+1}^{i+n-1} a_{j}+a_{i+n}\right)$

$\tilde{S}_{i j}$ denotes the large-scale strain rate tensor. $\tau_{i j}$ represents the subgrid scale stress in LES. $\Pi$ is the subgrid scale energy flux. In the large-scale kinetic energy equation (16), only the subgrid scale energy flux term is responsible for the scale-to-scale energy transfer. Positive $\Pi$ implies that the turbulent kinetic energy is transferred from the large scales to the smaller scales and minus $\Pi$ indicates that the turbulent kinetic energy is extracted from the small scales and transferred to the larger scales.

Fig. 8 shows the ensemble-averaged subgrid scale kinetic energy flux of different filter width including the total energy flux (Fig. 8(a)), the positive energy flux (Fig. 8(b)) and the minus energy flux (Fig. 8(c)). These energy fluxes keep decreasing in the large-scale range for present two jet flows. It can also be found that no matter for positive or minus energy flux, the energy flux of combustion jet flow is always larger than that of non-combustion jet flow. This phenomenon indicates that the occurrence of com- 

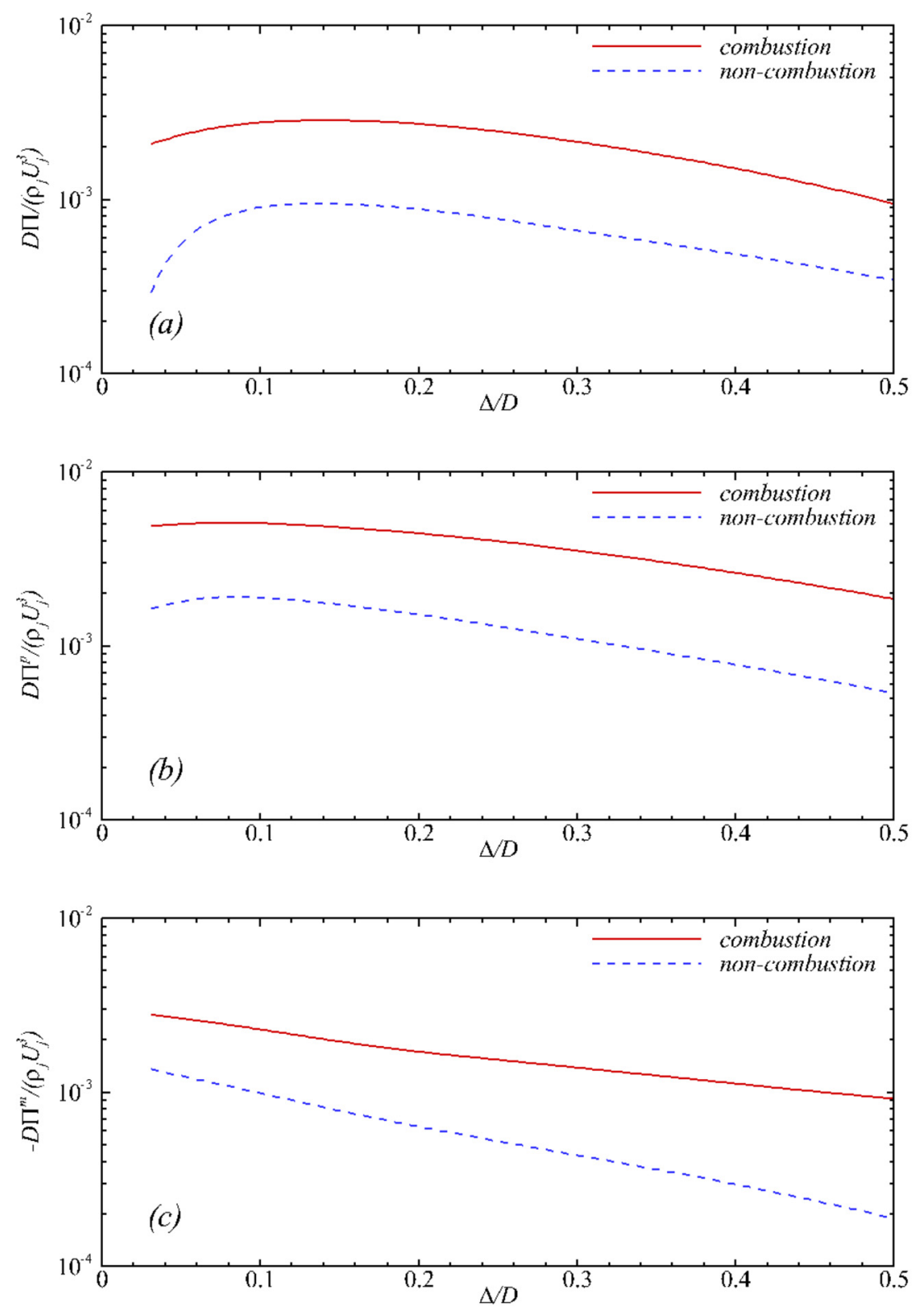

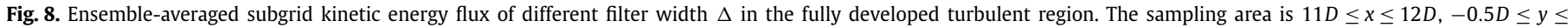
$0.5 D,-0.5 D \leq z \leq 0.5 D$. (a) Total energy flux $\Pi ;(b)$ positive energy flux $\Pi^{p} ;(c)$ minus energy flux $\Pi^{m}$.

bustion strengthens simultaneously both channels of the kinetic energy cascade.

\section{Conclusions}

In this paper, two direct numerical simulations of supersonic round turbulent hydrogen jets (with and without combustion) are conducted to investigate the effects of combustion on the characteristics of supersonic turbulence jet. Through analysis and comparison of the two cases, we can obtain the following conclusions:

(1) Compared with the non-combustion jet flow, the combustion jet has more complete coherent vortex rings in the initial stage. In the early stage, the vortex rings of non-combustion jet flow are helical, and the vortex rings of combustion jet flow are axisymmetric.

(2) Comparison with non-combustion one, the transition is significantly delayed for combustion jet flow. There are less vortex filament structures in the combustion jet, and the scales of the vortex filaments are coarser.

(3) The occurrence of combustion results in a $38 \%$ decrease in the peak of turbulent kinetic energy.

(4) In addition, the positive and minus energy fluxes of combustion jet flows are always larger than that of non-combustion jet flow.

\section{Declaration of competing interest}

There is no conflict of interest.

\section{Acknowledgements}

This work was supported by NSFC Projects (Nos. 91852203, 11472278), the National Key Research and Development Program of China (2016YFA0401200), Science Challenge Project (TZ2016001), and Strategic Priority Research Program of Chinese Academy of Sciences (Grant No. XDA17030100, XDC01000000). The authors 
thank National Supercomputer Center in Tianjin (NSCC-TJ), and National Supercomputer Center in GuangZhou (NSCC-GZ) for providing computer time.

\section{References}

[1] C. Zhang, J. Chang, S. Feng, J. Ma, J. Zhang, W. Bao, Pressure rising slope variation accompanying with combustion mode transition in a dual-mode combustor, Aerosp. Sci. Technol. 68 (2017) 370-379.

[2] L.q. Li, W. Huang, L. Yan, Mixing augmentation induced by a vortex generator located upstream of the transverse gaseous jet in supersonic flows, Aerosp. Sci. Technol. 68 (2017) 77-89.

[3] R.J. Weber, J.S. MacKay, An Analysis of Ramjet Engines Using Supersonic Combustion, NASA TN 4386, 1958.

[4] C. Segal, The Scramjet Engine: Processes and Characteristics, Cambridge University Press, 2009.

[5] T. Poinsot, D. Veynante, Theoretical and Numerical Combustion, RT Edwards, Inc., 2005.

[6] K.M. Saqr, M.M. Sies, M.A. Wahid, Numerical investigation of the turbulencecombustion interaction in nonpremixed CH4/air flames, Int. J. Appl. Math. Mech. 5 (2009) 69-79

[7] S. Lu, J. Fan, K. Luo, High-fidelity resolution of the characteristic structures of a supersonic hydrogen jet flame with heated co-flow air, Int. J. Hydrog. Energy 37 (2012) 3528-3539.

[8] T. Jin, K. Luo, S. Lu, J. Fan, Direct numerical simulation of a supersonic lifted hydrogen jet flame: a priori study on combustion models, Acta Astronaut. 109 (2015) 52-64.

[9] T. Jin, K. Luo, S. Lu, J. Fan, Analysis of conditional statistics of a supersonic jet flame in heated coflow via direct numerical simulation, Acta Astronaut. 134 (2017) 179-188

[10] K. Luo, J. Yang, Y. Bai, J. Fan, Large eddy simulation of turbulent combustion by a dynamic second-order moment closure model, Fuel 187 (2017) 457-467.

[11] D.H. Wacks, N. Chakraborty, M. Klein, P.G. Arias, G.I. Hong, Flow topologies in different regimes of premixed turbulent combustion: a direct numerical simulation analysis, Phys. Rev. E 1 (2016) 083401.

[12] W.G. Gottenberg, D.R. Olson, H.W. Best, Flame quenching during high pressure high turbulence combustion, Combust. Flame 7 (1963) 9-16.

[13] R.S. Barlow, J.H. Frank, Effects of Turbulence on Species Mass Fractions in Methane/Air Jet Flames, Elsevier, 1998, pp. 1087-1095.

[14] I.O. Moen, M. Donato, R. Knystautas, J.H. Lee, Flame acceleration due to turbulence produced by obstacles, Combust. Flame 39 (1980) 21-32.

[15] Z. Bo, Q. Li, H. Yong, P. Petersson, Z. Li, M. Aldén, X.S. Bai, Visualization of multi-regime turbulent combustion in swirl-stabilized lean premixed flames, Combust. Flame 162 (2015) 2954-2958.

[16] H.M. Li, G.X. Li, Y.H. Jiang, L. Li, F.S. Li, Flame stability and propagation characteristics for combustion in air for an equimolar mixture of hydrogen and carbon monoxide in turbulent conditions, Energy 157 (2018) 76-86.

[17] H. Fellouah, C.G. Ball, A. Pollard, Reynolds number effects within the development region of a turbulent round free jet, Int. J. Heat Mass Transf. 52 (2009) 3943-3954.

[18] N.R. Panchapakesan, J.L. Lumley, Turbulence measurements in axisymmetric jets of air and helium. Part 1. Air jet, J. Fluid Mech. 246 (1993) 197-223.
[19] H.J. Hussein, S.P. Capp, W.K. George, Velocity measurements in a highReynolds-number, momentum-conserving, axisymmetric, turbulent jet, J. Fluid Mech. 258 (1994) 31-75.

[20] Z. Wang, P. He, Y. Lv, J. Zhou, J. Fan, K. Cen, Direct numerical simulation of subsonic round turbulent jet, Flow Turbul. Combust. 84 (2010) 669-686.

[21] J. Mi, D.S. Nobes, G.J. Nathan, Influence of jet exit conditions on the passive scalar field of an axisymmetric free jet, J. Fluid Mech. 432 (2001) 91-125.

[22] R.M.C. So, J.Y. Zhu, M.V. Ötügen, B.C. Hwang, Some measurements in a binary gas jet, Exp. Fluids 9 (1990) 273-284.

[23] E.W. Grandmaison, D.E. Rathgeber, H.A. Becker, Some characteristics of concentration fluctuations in free turbulent jets, Can. J. Chem. Eng. 60 (1982) 212-219.

[24] C.D. Richards, W.M. Pitts, Global density effects on the self-preservation behaviour of turbulent free jets, J. Fluid Mech. 254 (1993) 417-435.

[25] B.J. Boersma, G. Brethouwer, F.T.M. Nieuwstadt, A numerical investigation on the effect of the inflow conditions on the self-similar region of a round jet, Phys. Fluids 10 (1998) 899-909.

[26] C. Bogey, C. Bailly, A study of the influence of the Reynolds number on jet selfsimilarity using large-eddy simulation, in: Direct and Large-Eddy Simulation VII, Springer, 2010, pp. 899-909.

[27] M.D. Slessor, M. Zhuang, P.E. Dimotakis, Turbulent shear-layer mixing: growthrate compressibility scaling, J. Fluid Mech. 414 (2000) 35-45.

[28] E. Ferdman, M.V. Oslash, u. $t$ gen, S. Kim, Effect of initial velocity profile on the development of round jets, J. Propuls. Power 16 (2000) 676-686.

[29] S.M.V. Rao, T. Ikeda, S. Asano, T. Saito, Far-field hot-wire measurements on free jet from complex supersonic nozzles, Appl. Therm. Eng. 118 (2017) 670-681.

[30] H. Xia, Turbulent jet characteristics for axisymmetric and serrated nozzles, Comput. Fluids 110 (2015) 189-197.

[31] S. Gordon, B.J. McBride, Computer Program for Calculation of Complex Chemical Equilibrium Compositions, Rocket Performance, Incident and Reflected Shocks and Chapman-Jouguet Detonations, NASA SP273, 1971.

[32] R. Design, CHEMKIN/CHEMKIN-PRO Theory Manual, CHEMKIN Software, No August, (2010) 1-360.

[33] C.R. Wilke, A viscosity equation for gas mixtures, J. Chem. Phys. 18 (1950) 517-519.

[34] R.B. Bird, W.E. Stewart, E.N. Lightfoot, Transport Phenomena, John Wiley \& Sons, New York, 1960, p. 413.

[35] X.L. Li, Y. Leng, Z.W. He, Optimized sixth-order monotonicity-preserving scheme by nonlinear spectral analysis, Int. J. Numer. Methods Fluids 73 (2013) 560-577.

[36] G. Strang, On the construction and comparison of difference schemes, SIAM J. Numer. Anal. 5 (1968) 506-517.

[37] Z. Ren, S.B. Pope, Second-order splitting schemes for a class of reactive systems, J. Comput. Phys. 227 (2008) 8165-8176.

[38] K. Kumaran, V. Babu, Investigation of the effect of chemistry models on the numerical predictions of the supersonic combustion of hydrogen, Combust. Flame 156 (2009) 826-841.

[39] J. Li, Z. Zhao, A. Kazakov, F.L. Dryer, An updated comprehensive kinetic model of hydrogen combustion, Int. J. Chem. Kinet. 36 (2004) 566-575.

[40] H. Liu, Z. Xiao, Scale-to-scale energy transfer in mixing flow induced by the Richtmyer-Meshkov instability, Phys. Rev. E 93 (2016) 053112. 